\title{
Fandom e industria culturale: la nascita del fandom di fantascienza negli Stati Uniti
}

\section{Paolo Bertetti ${ }^{1}$}

Recibido: 2017-01-19

Enviado a pares: 2017-02-14

DOI: $10.5294 /$ pacla.2017.20.4.11
Aprobado por pares: 2017-05-15

Aceptado: 2017-05-30

\section{Para citar este artículo / to reference this article / para citar este artigo}

Bertetti, P. (2017). Fandom e industria culturale: la nascita del fandom di fantascienza negli Stati Uniti. Palabra Clave 20(4), 1142-1160. DOI: 10.5294/pacla.2017.20.4.11

\section{Abstract}

Già da tempo è stato evidenziato come le attività di fandom siano una realtà complessa, luogo elettivo di pratiche grassroots di creatività e resistenza, ma anche oggetto di colonizzazione e cooptazione da parte dell'industria culturale, in una dinamica nella quale gli stessi fan si pongono come co-artefici. Si tratta di un elemento che è evidente nelle politiche dei franchising transmediali e nella cult-testualità televisiva contemporanei, ma che si può ritrovare in forme diverse nella storia del fandom fin dalle sue origini. Ciò, almeno, è vero per il fandom legato alla letteratura di fantascienza, che rappresenta a riguardo un caso sicuramente particolare: basti pensare alla quantità di fan che, fin dagli anni'40, sono diventati professionisti, come scrittori, critici, curatori e persino come editori. La stessa nascita di un fandom organizzato di fantascienza si deve, almeno in parte, all'attività di promozione "dall'alto" ad opera dell'industria culturale. In particolare, il nostro articolo analizza - attraverso lo studio della letteratura e la ricerca di archivio - il ruolo attivo svolto da Hugo Gernsback (editore di Amazing Stories, prima rivista di SF al mondo) nella nascita del fandom di fantascienza negli Stati Uniti: per la divulgazione del nuovo genere e la promozione delle pro-

1 orcid.org/0000-0001-5510-1405. Università degli Studi di Siena, Italia. bertetti@unisi.it 
prie riviste, egli infatti non solo favorì la nascita di gruppi di appassionati, pubblicando nella pagina della posta gli indirizzi dei lettori e divulgando notizie e informazioni sulle riunioni e i club, ma arrivò a fondare egli stesso una Science Fiction League, con sedi in numerose città degli Stati Uniti e persino in Inghilterra.

\section{Parole chiave}

Fantascienza; industria culturale; audience partecipanti; cultura di massa; letteratura popolare (Fonte: Thesaurus Unesco). 


\section{Fandom y la industria cultural: el nacimiento del fandom de la ciencia ficción en Estados Unidos}

\section{Resumen}

Desde hace tiempo se evidenció cómo la actividad del fandom es una realidad compleja, un lugar electivo de prácticas grassroot de creatividad y resistencia, pero también objeto de colonización y cooptación por parte de la industria cultural, en una dinámica en la que los mismos aficionados se posicionan como cocreadores. Se trata de un elemento evidente en las políticas de franquicias transmedia y en la textualidad televisiva contemporánea de culto, pero que se puede reencontrar de diversas maneras a lo largo de la historia del fandom y desde sus orígenes. Esto, por lo menos, es cierto en el caso del fandom relacionado con la literatura de ciencia ficción, que representa un caso sin duda particular: basta pensar en la cantidad de aficionados que desde los años 40 se han convertido en profesionales, como escritores, críticos, investigadores e incluso editores. El mismo nacimiento de un fandom organizado de ciencia ficción se debe, al menos en parte, a la actividad de promoción realizada "desde arriba" por la industria cultural. En particular, nuestro artículo analiza, mediante el estudio de la literatura y la investigación de archivo, el papel activo de Hugo Gernsback (editor de Amazing Stories, primera revista de SF del mundo) en el nacimiento del fandom de ciencia ficción en Estados Unidos: para la divulgación del nuevo género y la promoción de las mismas revistas, Gernsback no solo favoreció el nacimiento de grupos de apasionados, publicando las direcciones de los lectores y divulgando noticias e información sobre reuniones y clubes, pero llegó incluso a fundar una Science Fiction League, con sedes en numerosas ciudades de Estados Unidos e incluso en Inglaterra.

\section{Palabras clave}

Ciencia ficción; industria cultural; audiencia participante; cultura de masas; literatura popular (Fuente: Tesauro de la Unesco). 


\section{Fandom and the Cultural Industry: The Origin of Science Fiction Fandom in the United States}

\section{Abstract}

It has long become evident that fandom activity is a complex reality, but also the object of colonization and co-optation by the cultural industry, in a dynamic where fans themselves are co-creators. It is evident in the policies of transmedia franchising and in contemporary cult television textuality, but which may be found in different ways throughout the history of fandom and since its origin. This, at the very least, is true in the case of fandom related to science fiction, which represents an undoubtedly particular case: just think of the number of fans who, since the 1940s, have become professionals, such as writers, critics, researchers and even editors. The very birth of an organized science fiction fandom is due, at least in part, to the promotional activity conducted by the cultural industry "from above." More specifically, our paper analyzes, through the study of literature and archive research, the active role of Hugo Gernsback (editor of Amazing Stories, the first SF journal in the world) in the origin of science fiction fandom in the United States: In order to make the new genre known and promote the journal, not only did Gernsback favor the creation of passionate groups, publishing the readers' addresses and divulging news and information about meetings and clubs, he also founded a Science Fiction League, with offices in numerous US cities and even in England.

\section{Keywords}

Science fiction; cultural industry; participant audience; mass culture; popular literature (Source: Unesco Thesaurus). 


\section{Fandom e a indústria cultural: o nascimento do fandom da ciência ficção nos Estados Unidos}

\section{Resumo}

Há muito que se evidenciou como a atividade do fandom é uma realidade complexa, um lugar eletivo de práticas grassroot de criatividade e resistência, mas também objeto de colonização e cooptação por parte da indústria cultural, em uma dinâmica na qual os mesmos aficionados se posicionam como cocriadores. Trata-se de um elemento evidente nas políticas de franquias de transmídia e na textualidade televisiva contemporânea de culto, mas que se pode reencontrar de diversas maneiras ao longo da história do fandom e desde suas origens. Isto, pelo menos, é certo no caso do fandom relacionado com a ciência ficção, que representa um caso sem dúvida particular: basta pensar na quantidade de fãs que, desde os anos 40 tornaram-se profissionais, como escritores, críticos, pesquisadores e inclusive editores. O próprio nascimento de um fandom organizado de ciência ficção se deve, pelo menos em parte, à atividade de promoção realizada "desde cima" pela indústria cultural. Particularmente, nosso artigo analisa, através do estudo da literatura e da pesquisa de arquivo, o papel ativo de Hugo Gernsback (editor de Amazing Stories, primeira revista de SF do mundo) no nascimento do fandom de ciência ficção nos Estados Unidos: para a divulgação do novo gênero e da promoção das mesmas revistas, Gernsback não só favoreceu o nascimento de grupos de apaixonados, publicando os endereços dos leitores e divulgando notícias e informação sobre reuniões e clubes, mas chegou inclusive a fundar uma Science Fiction League, com sedes em inúmeras cidades dos Estados Unidos e inclusive na Inglaterra.

\section{Palavras-chave}

Ciência ficção; indústria cultural; audiência participante; cultura de massas; literatura popular (Fonte: Tesauro da Unesco). 
La tradizione dei Fandom Studies, ormai più che ventennale, ha da tempo evidenziato come le attività di fandom siano una realtà complessa, insieme luogo elettivo di pratiche grassroot di creatività e resistenza ed oggetto di colonizzazione e cooptazione da parte dell'industria culturale, in una dinamica nella quale gli stessi fan si pongono come co-artefici. Questo rapporto, al centro tra l'altro di quella "cultura convergente" delineata da Henry Jenkins (2006) nel volume omonimo, è evidente nelle politiche dei media franchising contemporanei, nei quali la circolazione dei contenuti attraverso diversi media avviene grazie al coinvolgimento degli utenti stessi sia a livello della loro promozione e distribuzione che - almeno in parte - della loro stessa produzione, a partire dalla creazione di user generated content fino alla cooptazione di elementi provenienti dal fandom nella produzione professionale (Jenkins, 2006a; Jenkins, 2006b).

Le ricerche più recenti son dunque ben distanti dall'idea, che aveva caratterizzato una prima fase dei fandom studies (Jenkins, 1992; Lewis, 1992), secondo la quale le pratiche di fandom erano tattiche oppositive all'industria culturale, alternative a un modello di fruizione passiva dei contenuti mediali. Abercrombie e Longhurst (1998) sottolineano piuttosto la sostanziale continuità esistente tra le attività dei pubblici generici, dei fan e di quelli che essi chiamano i "petty producers", vale a dire i produttori amatoriali di testi mediali. All'interno del paradigma "spettacolo/performance" proposto dai due autori, come sintetizza Agnese Vellar (2015), "le pratiche partecipative non sono dunque ne' una forma di devianza ne' di resistenza, ma piuttosto uno dei differenti gradi di coinvolgimento, partecipazione e professionalizzazione in relazione ad un testo o genere mediale" (p. 76).

Numerosi studiosi, in particolare, hanno analizzato il "fandom come lavoro" (Mel \& Condis, 2014) e il valore economico che le attività di fandom rivestono per le industrie culturali, vendendole sia come lavoro gratuito oggetto di sfruttamento da parte dei media per finalità di promozione (Terranova, 2000), sia apprendistato per l'ingresso nel mondo del professionismo (Baym \& Burnett, 2009). In genere, questi studi hanno posto il fenomeno in relazione, non esclusiva ma comunque stretta, con la diffusione delle tecnologie digitali e del Web 2.0. Pur non volendo negare l'importanza delle nuove tecnologie nell'evoluzione del fandom e del suo rapporto con 
le industrie dell' intrattenimento, riteniamo che tale permeabilità tra amatorialità e industrie dei media sia una caratteristica che si possa ritrovare in forme diverse nella storia del fandom fin dalle sue origini.

Ciò è quanto meno vero per il fandom della fantascienza, in particolare quello legato alla letteratura, che sarà oggetto di questo articolo. A questo riguardo, esso costituisce un caso sicuramente particolare; come già osservava Edward James (1994) nella sua storia della fantascienza, infatti, "non c'è nessun ramo della letteratura dove il contatto tra lettore e scrittore sia stato così stretto e l'interazione così forte e duratura" (p. 130): basti pensare alla quantità di fan che, fin dagli anni ' 30 e ' 40 , sono diventati professionisti, come scrittori, critici, curatori e persino come editori. Per fare un esempio, alle riunioni di quello che è forse il fan club più famoso del periodo, quello dei Futurians, nato nel 1937 a New York, partecipavano alcuni di quelli che sarebbero diventati gli autori di punta della Golden Age della letteratura fantascientifica: Isaac Asimov, Cyril Kornbluth, Robert Lowndes, Frederick Pohl, Richard Wilson, Damon Knight, Judith Merril, ma anche un illustratore come Hannes Box e persino quello che sarebbe diventato uno dei maggiori editori specializzati nel genere, Donald $\mathrm{A}$. Wollheim (Pohl, 1979, pp. 65-95; Knight, 1977). All'altro capo degli States, la Los Angeles Science Fiction Society poteva vantare figure come il futuro autore delle Cronache Marziane, Ray Bradbury, il mago degli effetti speciali Ray Harryhausen e l'agente letterario Forrest J Ackerman.

Fin dall' 'inizio, quindi, il fandom fantascientifico ha svolto un ruolo di intermediazione fra consumo e produzione culturale, costituendo un trampolino per giovani talentuosi con velleità professionistiche. Tuttavia, il rapporto tra industria culturale e fandom fantascientifico è stato in realtà, fin dalle sue origini, assai più complesso: la stessa nascita di un fandom organizzato di fantascienza si deve infatti, almeno in parte, all'attività di promozione "dall'alto" ad opera dell' industria culturale, ed in particolare al ruolo attivo svolto dall'editore Hugo Gernsback.

Gernsback è unanimemente considerato il "padre" della fantascienza. Si deve infatti a lui la pubblicazione della prima rivista interamente de- 
dicata al genere, Amazing Stories (storie sorprendenti), apparsa nel mese di aprile 1926. Gernsback era un elettrotecnico lussemburghese immigrato negli Stati Uniti, dove fondò nel 1908 una delle prime riviste tecniche di radiocomunicazioni, Modern Electrics (diventata poi The Electrical Experimenter e successivamente Science and Inventions). A partire dal numero di aprile 1911 della rivista, incominciò a pubblicare a puntate il suo romanzo avveniristico Ralph124C41+, e successivamente continuò a pubblicare regolarmente "narrativa scientifica" (Scientific Fiction), alla quale dedicò interamente il numero di aprile 1923. Visto il successo Gernsback ruppe gli indugi e diede finalmente vita ad Amazing, la prima rivista interamente dedicata al genere che egli battezzò "Scientifiction"; con tale termine spiega nell'editoriale del primo numero - egli intendeva "storie sul tipo di quelle che scrivevano Jules Verne, H.G. Wells, Edgar Allan Poe, vale a dire vicende in cui alla trama romanzesca si intrecciano fatti scientifici e visioni profetiche del futuro" (citato in Sadoul, 1975, p. 60).

Prima di allora racconti e romanzi di fantastico scientifico (il cosiddetto scientific romance), oltre che sulle riviste di Gernsback, erano regolarmente ospitati sulle riviste pulp di inizio novecento, in particolare su Argosy, All Story Magazine, The Cavalier del gruppo editoriale Munsey, e anche a partire dal 1923 - sul primo pulp interamente dedicato alla letteratura fantastica, Weird Tales che, sebbene prevalentemente dedicato al Weird e all'Horror, non mancava di ospitare fantasie scientifiche. Quando Gernsback inizia a pubblicare Amazing Stories, poteva dunque già contare su un pubblico consistente e soprattutto appassionato (Moskowitz, 1990, p. 18).

Già nell'editoriale del terzo numero, Gernsback afferma di "essere sommerso dal grandissimo numero di lettere ricevute $\mathrm{da}$ - possiamo chiamarli 'fan della scientifiction'? - che sembrano assai ben disposti verso questo tipo di letteratura" (citato in Moskowitz, 1990, p. 18). Gernsback si rese subito conto del "capitale emozionale" (come diremmo ora) che aveva in mano e dell'utilità a fini promozionali di coinvolgere e fidelizzare i fan, dando loro spazio e importanza. Nel numero del gennaio 1927 di Amazing, egli inaugurò una rubrica di "Discussioni", nella quale pubblicava le lettere dei lettori. Gunn (1975, p. 124) sottolinea il ruolo che, attraverso 
la pagina della posta, ebbero i fan nell'orientare alcune scelte di Amazing e lo stesso sviluppo della fantascienza, promuovendo alcune storie e precipitandone altre nella polvere. Gunn (1975, p. 124) osserva anche come lo sviluppo del fandom di fantascienza (e più in generale, aggiungeremmo noi, della letteratura fantastica) sia stato un unicum nel campo della narrativa: sebbene anche altri generi, come il western o il poliziesco, avessero i loro patiti difficilmente qualcuno di essi si sarebbe sentito parte di una comunità in base ai propri gusti e tanto meno avrebbe dato a origine a incontri locali, regionali, nazionali e perfino internazionali. ${ }^{2}$ Questo era forse maggiormente vero all'epoca in cui scriveva Gunn piuttosto che non adesso, in un'epoca di generalizzata esplosione delle pratiche di fandom, ma è comunque indubbio che ancor oggi è proprio nell'ambito fantastico che si situano la maggior parte dei culti mediali (come osservavano già Gwenliann \& Pearson, 2004). In ogni caso andrà quanto meno riconosciuto come sia stato proprio in ambito fantascientifico che nacque il primo fandom organizzato.

I motivi di questa peculiarità vanno sicuramente ricercati in una serie di nessi che legano alcuni aspetti caratteristici del nuovo genere ad alcune variabili individuali e sociali: la fantascienza è un genere letterario che seppure popular - è talvolta di non facile consumo e soprattutto richiede una capacità di "sospensione dell'incredulità" e attitudini fruitive non sempre rintracciabili nel pubblico generico; esso è per sua natura destinato ad essere un genere di culto, apprezzato fanaticamente da pochi. Ciò doveva a maggior ragione essere vero agli arbori della sua storia; come ricorda Frederick Pohl (1978),

nei primi anni '30 essere lettore di fantascienza era una cosa triste e solitaria. Non eravamo in molti, e non avevamo modo di incontrarci l'un l'altro per parlarci. [...] avevamo una permanente sensazione di essere soli in un mondo ostile. Le orde dei non-adepti erano semplicemente disinteressate alla fantascienza e la consideravano ridicola" (pp. 17-18).

2 Anche se è vero, continua Gunn, che ci possono essere dei fan club legati a un singolo scrittore o a un personaggio (si pensi ai fans di Sherlock Holmes e a società come "Gli irregolari di Baker Street"). 
A tale incomprensione del pubblico, corrispondeva da parte del fan la convinzione di essere una sorta di élite del pensiero; come osserva James (1994), commentando alcune fonti dell'epoca, "i fans della fantascienza si mettevano insieme non tanto perché amassero leggere fantascienza, ma piuttosto perché avevano una comune visione del posto dell'umanità nell'universo [...] che li distingueva, a lor sentire, dal monotono mondo della gente comune che li circondava" (p. 132).

Se dunque già allora, come succederà poi nei fandom mediali successivi (Jenkins, 1998; Scaglione, 2006), la molla che spingeva il fan all'attivismo è l'isolamento sociale e culturale e il desiderio di condivisone della propria passione, alla quale si aggiunge una visione quasi religiosa del proprio oggetto di culto, è tuttavia assai probabile che lo sviluppo di un fandom organizzato nell'ambito della letteratura di fantascienza e non in altri generi si debba in parte notevole proprio all'attivismo di Gernsback, il quale per la promozione del nuovo genere, favorì la nascita di gruppi di appassionati, non solo dando spazio alle lettere dei fan (in fondo, come osserva Ashley, 1975, la pagina della posta non era una novità nelle riviste pulp; Weird Tales, ad esempio, aveva The Eyrie), ma soprattutto decidendo di pubblicare, alla fine di ogni lettera, l'indirizzo completo dello scrivente, in modo che potessero entrare in contatto tra loro e conoscersi. Moskowitz (1990), nella sua ricognizione sulle origini del fandom di fantascienza statunitense, considera anzi questa iniziativa la causa fondamentale, se non unica, dello sviluppo di un fandom di fantascienza organizzato.

Del resto, lo stesso Gernsback (1929) lo rivendicava con orgoglio:

La rubrica Discussioni su Amazing Stories [...] è per noi un punto di merito. Essa fa sì che i lettori possano corrispondere tra loro e rende assai facile la formazione di club dei lettori. Solo che devono essere i lettori a fare tale lavoro. ${ }^{3}$ (p. 432)

Infatti, nonostante la redazione non potesse farsi direttamente carico dell'organizzazione, la rivista era interessata alla fondazione di club: "de-

3 Amazing Stories Quarterly, estate 1929, p. 432. Per le citazioni tratte dai pulp ho attinto dal ricco Fondo Riccardo Valla in deposito presso il Mufant. Museo della Fantascienza e del Fantastico di Torino. 
vono essere gli stessi lettori che ci scrivono ad organizzare il club, sapendo che noi siamo interessati all'iniziativa e saremo lieti, quando essa avrà preso forma, di dare spazio sulle nostre pagine ai suoi progressi" (Gernsback, 1929, p. 432). In effetti, uno Science Correspondence Club era già nato proprio attraverso le pagine di Amazing Stories alcuni mesi ad opera di due fans di Chicago, Walter Dennis e Sidney Gerson, grazie a uno scambio di lettere con un altro fan di New York, George Lasky. L'idea dei due era di creare anche dei gruppi locali per discutere di scienza e fantascienza, allo scopo di diffondere "la scienza e il pensiero scientifico tra i profani del mondo" (citato in Moskowitz, 1990, p. 22); ${ }^{4}$ per far conoscere la propria iniziativa si servirono proprio degli indirizzi dei lettori pubblicati sulla pagina della posta delle riviste di Gernsback. L'associazione si allargò ben presto a livello nazionale, cooptando altri club di appassionati locali, e arrivò a produrre un proprio bollettino, The Comet, curato dallo stesso Dennis e da Raymond Palmer, un giovane fan che in seguito avrebbe svolto una luminosa carriera professionistica nel mondo dei pulp, diventando direttore della stessa Amazing. Il primo numero di The Comet fu pubblicato nel maggio 1930 e consisteva di dieci pagine ciclostilate; l'intento originario era di fare divulgazione scientifica, ma via via l'interesse principale divenne la narrativa fantascientifica (Nadis, 2013). Ben presto arrivò anche la prima rivista amatoriale dedicata alla fantascienza, The Planet, pubblicata tra giugno e novembre del 1930 da un nuovo gruppo di appassionati di New York, The Scienceers.

Gernsback — che nel frattempo, perso il controllo di Amazing Stories, aveva fondato una nuova casa editrice e varato nel 1929 ben due nuove riviste di fantascienza, Air Wonder Stories e Science Wonder Stories, poi riunite l'anno successivo in Wonder Stories — pubblicò la notizia sul numero di maggio 1930 di Science Wonder Stories, offrendo il suo "entusiastico supporto" all'iniziativa. In effetti, dalle pagine delle sue nuove riviste, il padre della fantascienza prese a incentivare sempre più le attività del fandom, dando loro visibilità nella pagina della posta di Science Wonder Stories, “The Reader Speaks", ma non solo: nello stesso numero del maggio 1930, ad esempio, l'editoriale era interamente dedicato a una "Settimana della fantascienza" organizzata da un fan dell'Indiana per promuoverne la conoscenza. In esso

4 Dall'editoriale anonimo "A brief history of the Red Cell Club", uscito sulla fanzine The Comet, Maggio 1930. 
Gernsback, che considerava sinceramente la fantascienza un potente veicolo di diffusione della cultura scientifica, mostra uno spirito proselitistico quasi missionario, che andava al di là del puro interesse per la promozione delle sue riviste. Scriveva, infatti:

La fantascienza è un fattore importante per rendere il mondo un posto migliore dove vivere, attraverso l'educazione del pubblico alle possibilità della scienza [...] se ogni uomo, donna, bambino e bambina venisse indotto a leggere science fiction, ne deriverebbe senza dubbio un considerevole beneficio per la comunità, in quanto il livello di istruzione si eleverebbe enormemente. (Gernsback, 1930, p. 1061)

L'editoriale continuava incoraggiando le attività dei fan e invitando " $\mathrm{i}$ veri amanti della fantascienza" a diventare pubblico attivo, facendo conoscere il genere attraverso l'organizzazione di letture pubbliche e altre iniziative.

Gernsback era assolutamente convinto dell'importanza di coinvolgere nella diffusione del nuovo genere i lettori più appassionati. Come ricorda Kyle (1993), un esponente di spicco del primo fandom, nel fare ciò egli era mosso da due diversi motivi: il primo era ovviamente quello di accrescere i propri lettori e quindi trarre guadagno. Il secondo era molto più illuminato: egli era appassionatamente convinto che quel nuovo tipo di letteratura, che la scientifiction fosse la letteratura ideale per la diffusione della conoscenza scientifica e tecnica. Nel fare ciò egli si presentava come una sorta di profeta, con un particolare interesse verso gli appassionati di scienza: nelle sue parole i lettori più affezionati erano "pionieri" di un futuro migliore basato sul potere della scienza, illuminati adepti di quello che veniva a configurarsi come una sorta di vero e proprio culto laico e tecnocratico.

Per incentivare tale coinvolgimento egli aveva già bandito, sul numero dell'autunno 1929 di Science Wonder Quarterly (supplemento trimestrale di Science Wonder Stories), un concorso con 500 dollari di premio destinati a coloro che, tra i lettori, avessero fatto di più per la diffusione della fantascienza e, nel numero della primavera 1930, in concomitanza con la "Settimana della fantascienza" annunciava la stampa di una serie di etichette promozionali di varie dimensioni che sarebbero stati inviate gratuitamente a tutti i lettori chi ne facevano richiesta per diffonderle e attaccarle in giro. 
Nonostante l'interesse che aveva da sempre mostrato verso le attività dei fans, Gernsback, come si è detto, aveva sempre preferito che questi ultimi si auto-organizzassero, limitandosi generalmente a dare spazio alla divulgazione delle loro iniziative sulle pagine delle proprie riviste. Fu soltanto alcuni anni dopo, e precisamente nel 1934, che egli decise di giocare un ruolo più attivo nell'organizzazione del fandom, al fine di poter meglio sfruttare le possibilità di promozione della fantascienza in generale e di fidelizzazione verso le proprie riviste in particolare. C’è da dire che nel frattempo era diventato direttore associato di Wonder Stories Charles Hornig, proveniente giovanissimo proprio dal mondo del fandom, dove aveva pubblicato un unico numero della propria fanzine The Fantasy Fan prima di venire cooptato nel professionismo dallo stesso Gernsback che ne aveva apprezzato il lavoro editoriale; forse tale esperienza potrebbe non essere stata ininfluente nella decisione di quest'ultimo di svolgere un ruolo più attivo nell'organizzare gli appassionati.

Fatto sta che nell'editoriale dell'aprile 1934 di Wonder Story Gernsback lancia la Science Fiction League, "un'organizzazione associativa non commerciale senza alcuna tassa o quota di iscrizione. Una associazione esclusivamente letteraria e scientifica per la promozione della letteratura scientifica in ogni lingua" (Gernsback, 1934a, p. 933). Il suo scopo era quello di "coordinare tutti coloro che sono interessati alla fantascienza in un unico gruppo internazionale” (p. 933) e Gernsback auspicava che essa potesse dare origine a livello locale a innumerevoli club di fantascienza sparsi in tutto il mondo. Wonder Stories sarebbe stato l'organo ufficiale dell'associazione. L'annuncio era accompagnato da uno stemma dell'associazione, disegnato da Frank R. Paul, che mostrava un'astronave sullo sfondo del pianeta terra (figura 1).

L'editoriale del numero successivo riportava i nomi del "comitato esecutivo" dell'associazione (oltre a Gernsback e Hornig, in veste di segretari, erano presenti scrittori di fantascienza di primo piano come Eando Binder, Edmond Hamilton, David Keller, Clark Ashton Smith e P. S. Miller, nonché uno dei primissimi e più conosciuti fan, Forrest $J$ Ackerman) ma soprattutto rivelava tutti i dettagli dell'operazione, finalizzata a "disseminare il cul- 


\section{Figura 1. L'emblema della science Fiction League (disegno di Frank R. Paul)}

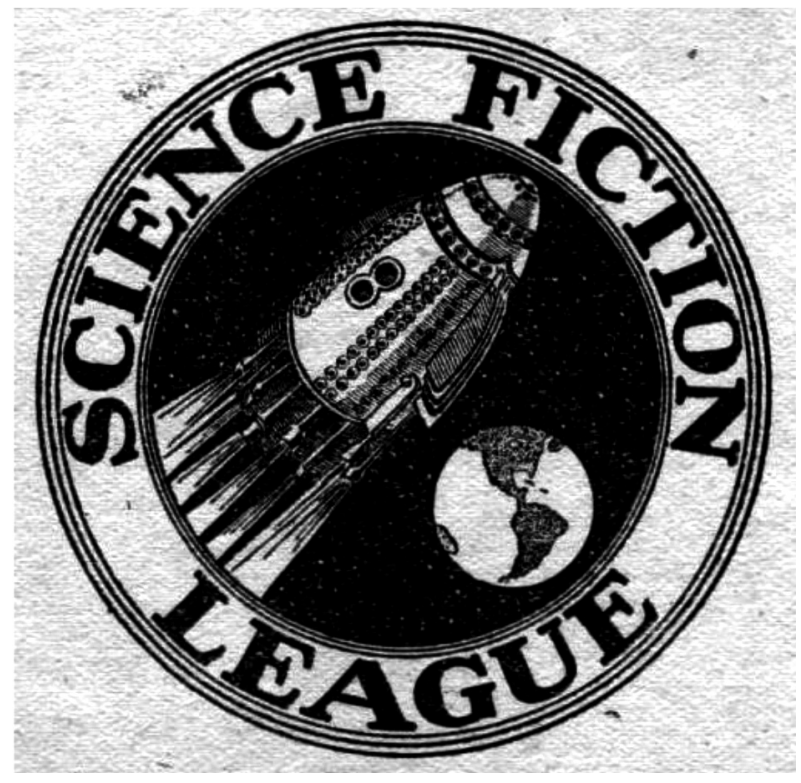

Fonte: Gernsback (1934a, p. 933).

to e l'arte della fantascienza" (Gernsback, 1934b, p. 1061), diffondendone con tutti i mezzi la conoscenza tra i possibili lettori e la sua diffusione sui diversi media (cinema, giornali, radio). L'iniziativa era senza scopo di lucro e non era finalizzata alla vendita di prodotti commerciali. Tuttavia essa forniva ai soci "a prezzo di costo più una piccola percentuale per le spese di gestione della League" (Gernsback, 1934, p. 1062)5 una serie di accessori ritenuti essenziali quali distintivi, carta da lettera, buste intestate e, all'atto dell'iscrizione, un certificato. In realtà, la Lega si limitava a coordinare e promuovere le iniziative delle diverse sezioni locali di cui si caldeggiava la costituzione e a suggerire una serie di possibili iniziative e strategie di successo; a tale scopo, a partire dal numero di giugno, una sezione di Wonder Stories venne interamente dedicata alle attività delle diverse sezioni, ospitando le discussioni e le proposte dei membri. L’accoglienza da parete dei

5 Ashley (2000), nella sua storia delle riviste di fantascienza riporta un numero leggermente inferiore, intorno al migliaio di iscritti. 
fan fu entusiastica e nei mesi successivi sorsero rapidamente numerose sezioni in tutto il territorio degli Stati Uniti e persino alcune in Inghilterra, in Irlanda, a Belfast, e addirittura una in Australia, a Sidney (Ashley, 1975, p. 44). Nelle grandi città del Nord America, anzi, erano spesso presenti più sezioni della League, che talvolta finivano di entrare in contrasto tra loro. Nel numero dell'aprile 1936 di Wonder Story, subito prima della forzata interruzione delle attività della League, un redazionale riferiva che gli iscritti erano quasi 1200 e le sezioni attivate negli Stati Uniti e all'estero erano 36.

Nonostante tutto questo movimento, tuttavia, l'impatto sulle vendite fu modesto. Coloro che si iscrivevano alla League erano in gran parte già acquirenti abituali della rivista; come osserva Ashley (2000), è difficile dire quanti di loro avessero effettivamente portato nuovi fan alla fantascienza e nuovi lettori a Wonder Stories, ma probabilmente non molti: secondo le sue stime, infatti, gli iscritti alla Lague non erano più del 3\% dei lettori totali, una percentuale in linea con quella dei lettori più attivi e partecipi di qualsiasi rivista.

Quello che è certo è che, nel corso del 1935, le vendite di Wonder Stories subirono una inesorabile flessione, che non venne contenuta nemmeno dalla diminuzione del prezzo di copertina; la rivista divenne prima bimestrale, poi con il succitato numero di aprile interruppe improvvisamente le pubblicazioni, Infine fu venduta alla Standard Magazines, che già pubblicava diverse riviste pulp di ambito prevalentemente poliziesco. Il nuovo editore, Leo Margolin, cambiò il nome della rivista in Thrilling Wonder Stories, inaugurando una linea editoriale diversa, incentrata su storie avventurose rivolte a un pubblico più giovane, e si disinteressò alla Science Fiction League.

Se da un punto di vista promozionale la Science Fiction League si rivelò dunque un fallimento, essa risultò tuttavia importantissima per la creazione di un fandom organizzato. Nonostante la sua forzata scomparsa, molte delle sezioni locali continuarono la loro attività, dando vita ad associazioni autonome alcune delle quali, come la Philadelphia Science Fiction Society, la Los Angeles Science Fantasy Society o la San Francisco Society, faranno la storia del fandom americano (James, 1995, p. 132). 
Come osserva Pohl (1978) raccontando nelle sue memorie le sue prime esperienze come fan e come membro della League:

Qualsiasi cosa la SFL abbia fatto per Gernsback, essa fece tantissimo per noi che praticavamo il solitario vizio della fantascienza. Essa ci fece uscire dal chiuso delle nostre stanze e ci condusse nel Fandom, portandoci direttamente a quelle specie di orge di gruppo che sono oggi le worldcon, dove centinaia di persone si impegnano nella celebrazione della fantascienza. (Pohl, 1978, p. 21)

Tre anni dopo la fine forzata della Science Fiction League, si tenne a New York la prima Worldcon, la convention mondiale di fantascienza; si trattò in realtà di un raduno di un numero limitato di fan statunitensi, ma il suo internazionalismo e l'idea stessa di porsi come un luogo di incontro la diverse realtà locali del fandom erano sicuramente un'eredità dell'organizzazione gernsbackiana.

Gernsback, come si è detto, assumeva a volte i toni di un profeta che si rivolgeva ai suoi proseliti, i fan lettori abituali della sue riviste e della fantascienza. Nel promulgare la sua visione della fantascienza come letteratura educativa, egli forniva ai fan delle motivazioni "forti” alla lettura di un genere spesso invece stigmatizzato: essi non erano più dei dropout, ma un'élite in possesso di una sensibilità tecnocratica e scientifica che avrebbe cambiato le sorti del pianeta.

Nella nascita del fandom di fantascienza in America, l'attivismo gernsbackiano è stato dunque importante, non soltanto per aver creato le condizioni e gli strumenti di aggregazione, ma anche per aver fornito un'ideologia capace di riunire appassionati altrimenti isolati, e in definitiva nel trasformare la fantascienza in un genere di culto, non solo da coltivare socialmente in comune ma anche da promuovere e diffondere attivamente - già ottant'anni fa — attraverso il lavoro appassionato dei fan.

Abbiamo già esposto in altra sede la nostra convinzione che certi fenomeni, come il transmedia storytelling (Scolari, Bertetti, \& Freeman, 2014), lungi dall'essere legati all'avvento delle nuove tecnologie, siano in realtà fenomeni di più lungo corso e che — mutatis mutandis — vi sia una sostanziale 
continuità nelle pratiche dell' industria culturale, o almeno in certi aspetti di essi. Lo stesso può forse dirsi di certe pratiche di convergenza tra produzione e consumo identificate spesso come caratteristiche del fandom mediale contemporaneo: la nascita del fandom fantascientifico in America, così fortemente caratterizzata dalla convergenza tra uno spontaneismo bottom up e un attivismo top down (Jenkins, 2006a), e la sua stessa natura di luogo di intermediazione tra consumo e produzione, nel quale il fan aspirante scrittore o editor era in grado di acquisire un capitale di competenze e di relazioni da spendere per approdare al professionismo (James, 1994, pp. 102-108), induce a ripensare visioni unidirezionali, forse eccessivamente semplificatrici, dell' industria culturale del novecento in favore di modelli più complessi di interazione tra produzione e consumo, a loro modo anticipatori, in nuce, di certi aspetti propri delle culture convergenti dei giorni nostri.

\section{Bibliografia}

Ashley, M. (1974). The history of science fiction magazines, Part one (19261935). London: New English Library.

Ashley, M. (2000). The time machine: The story of the science-fiction pulp magazines from the beginning to 1950. Liverpool: Liverpool University Press.

Baym, N. K., \& Burnett R. (2009). Amateur experts: International fan labor in Swedish independent music. International Journal of Cultural Studies, 12(5), 433-449. http://dx.doi. org/10.1177/136207877909337857

Gernsback, H. (1930). The science fiction week, Science Wonder Stories, 1(12), p. 1061.

Gernsback, H. (1934a). The science fiction league. An announcement, Science Wonder Stories, 5(9), p. 933.

Gernsback, H. (1934b). The science fiction league. Wonder Stories, 5(10), 1061-1065. 
Gunn, J. (1975). Alternate worlds: An illustrated history of science fiction. Englewood Cliffs: Prentice-Hall.

Gwnllian, J. S., \& Pearson, R. E. (eds.) (2004). Cult television. Minneapolis: University of Minnesota Press.

Hoffman, J.F. (2001). A briefhistory of the Red Cell Club. Blood Cells, Molecules, and Diseases, 27(1), 54-56.

Knight, D. (1977). The futurians. New York: John Day.

Kyle, D. (1993). The science fiction league. Mimosa, 14, 17-24. Disponibile da http://www.jophan.org/mimosa/m14/kyle.htm

James, E. (1995). Science fiction in the twentieth century. Oxford: Oxford University Press.

Jenkins, H. (1992). Textual poachers: Television fans \& participatory culture. Studies in culture and communication. New York: Routledge.

Jenkins, H. (2006a). Convergence culture: Where old and new media collide. New York: New York University Press.

Jenkins, H. (2006b). Fans, bloggers, and gamers: Exploring participatory culture. New York: New York University Press.

Lewis, L. A. (Ed.). (1994). The adoring audience. Oxford: Oxford University Press.

Stanfill, M., e Condis, M. (Eds.). (2014). Fandom and/as labor. Transformative Works and Cultures, 15. http://dx.doi.org/10.3983/ twc.2014.0593

Moskowitz, S. (1954). The immortal storm: A history of science fiction fandom. Atalanta: ASFO Press. 
Moskowitz, S. (1990). The origins of science fiction fandom: A reconstruction. Foundation, 5-25.

Nadis, F. (2013). The man from mars: Ray Palmer's amazing pulp journey. London: Penguin.

Pohl, F. (1979). The way the future it was: A memoir. New York: Ballantine/Del Rey.

Reid, A. R. (2009). Fan studies. In M. Bould, A. M. Butler, A. Roberts, e S. Vint (eds.), The Routdledge companion to science fiction (pp. 204214). Milton Park: Routledge.

Sadoul, J. (1973). Histoire de la science-fiction moderne. Paris: Albin Michel.

Scolari, C., Bertetti, P., e Freeman, M. (2014). Transmedia archaeology. Basingstoke: Palgrave.

Terranova, T. (2000). Free labor: Producing culture for the digital economy. Social Text, 18, 33-58. http://dx.doi.org/10.1215/01642472-182_63-33

Vellar, A. (2015). Le industrie culturali e i pubblici partecipativi: dalle comunità di fan ai social media. Roma: Aracne. 